\section{Nonalcoholic fatty liver disease is a risk factor for CKD in patients with type 2 diabetes}

The proportion of patients with type 2 diabetes who are on dialysis is steadily increasing. Early treatment of chronic kidney disease (CKD) delays the onset of end-stage renal disease in these individuals; therefore, the identification of risk factors for CKD is imperative. Targher et al. investigated whether nonalcoholic fatty liver disease (NAFLD), which is thought to affect $70-75 \%$ of patients with type 2 diabetes, is a risk factor for CKD in this population.

The authors enrolled 1,827 outpatients from the Valpolicella Heart Diabetes Study who had type 2 diabetes, normal or near-normal kidney function and no overt proteinuria at baseline. During 6.5 years of follow-up, 547 of the 1,760 participants who attended routine medical examinations developed incident CKD. Patients with NAFLD, as diagnosed by patient history and liver ultrasonography, were at increased risk of developing CKD (hazard ratio 1.69; $P<0.001$ ). Adjustments for confounders such as sex, age, BMI, waist circumference, blood pressure, smoking, glycosylated hemoglobin level, blood lipids, baseline estimated glomerular filtration rate, microalbuminuria and medication use did not substantially modify the correlation (hazard ratio 1.49; $P<0.01$ ).

CKD and NAFLD share many risk factors; however, the data suggest that NAFLD confers an additional risk of CKD, beyond that which would be expected from the common risk factors alone. Systemic release of proinflammatory mediators from the liver (e.g. C-reactive protein, fibrinogen and plasminogen activator inhibitor 1) and worsening of insulin resistance could be among the major underlying mechanisms by which NAFLD, especially in its necro-inflammatory form, increases the risk of CKD.

Original article Targher G et al. (2008) Increased risk of CKD among type 2 diabetics with nonalcoholic fatty liver disease. J Am Soc Nephrol 19: 1564-1570

\section{Effect of raloxifene on bone mineral density is enhanced in women with stage 3 CKD}

Although chronic kidney disease (CKD) is associated with an increased likelihood of osteoporosis, the efficacy and safety of therapies for osteoporosis in women with CKD remain unclear.

Ishani et al. analyzed data from 7,316 postmenopausal women with osteoporosis who participated in the international, randomized, placebo-controlled Multiple Outcomes of Raloxifene Evaluation (MORE) trial. The trial excluded women with a serum creatinine level $>230 \mu \mathrm{mol} / \mathrm{l}$. Raloxifene, a selective estrogen receptor modulator, was administered at a dose of $60 \mathrm{mg}$ per day or $120 \mathrm{mg}$ per day.

Bone mineral density measurements after 3 years of follow-up were obtained for 5,675 $(78 \%)$ women. Among those who received placebo, declining baseline Cockcroft-Gaultestimated creatinine clearance was associated with a trend toward decreased bone mineral density at the femoral neck $(P=0.091)$; however, among the raloxifene-treated patients, decreased creatinine clearance was associated with increased bone mineral density $(P=0.012)$. Raloxifene use was also associated with a decreased risk of vertebral fractures, but this association was not influenced by baseline kidney function. Raloxifene treatment did not affect the rate of nonvertebral fractures. Finally, there was no correlation between the total number of serious adverse events and raloxifene use at any level of kidney function.

The effect of raloxifene on bone mineral density at the hip seems to be heightened in women with reduced renal function, although this finding might apply just to those with mild to moderate CKD since only 55 patients in the MORE study had stage $4 \mathrm{CKD}$.

Original article Ishani A et al. (2008) The effect of raloxifene treatment in postmenopausal women with CKD. J Am Soc Nephrol 19: 1430-1438

\section{Risk of bleeding after kidney biopsy is not increased in individuals with amyloidosis}

Kidney biopsy is an important diagnostic tool in amyloidosis; however, an increased risk of post-biopsy bleeding has been suggested to occur in patients with this condition. A morecareful biopsy technique and increased observation might, therefore, be required, but these ideas have not been confirmed in large studies. Soares et al. have attempted to address this lack of data. 